Proc. Indian Acad. Sci. (Chem. Sci.), Vol. 102, No. 5, October 1990, pp. 613-628,

(C) Printed in India.

\title{
Laser Raman spectroscopy of colloidal semiconductor photosensitizers
}

\author{
S UMAPATHY and R E HESTER* \\ Chemistry Department, University of York, York YO1 SDD, UK
}

\begin{abstract}
Colloidal semiconductor photosensitizers have been used as heterogeneous catalysts for the chemical solar energy conversion processes. The use of Raman spectroscopy to address the problems related to colloidal semiconductors and photosensitization reactions is reviewed. In particular, the adsorption characteristics of various secondary sensitizers and their electron-transfer reactions are studied using resonance Raman and time-resolved resonance Raman spectroscopy. Finally, the importance of the quantum size effect of colloidal semiconductors in relation to their catalytic effects is presented with particular reference to their influence on photosensitizing properties.
\end{abstract}

Keywords. Laser Raman spectroscopy; colloidal semiconductor photosensitizers; heterogeneous catalysts; chemical solar energy conversion processes.

\section{Introduction}

Photosplitting of water into hydrogen and oxygen using heterogeneous catalysts has been the objective of many of the chemical solar energy conversion processes. In particular, there has been considerable activity in developing suitable colloidal semiconductors to sensitize and to mediate the water-splitting redox reaction (Grätzel 1985). In spite of the rapid progress made in this area of research, there are still many features that require further investigation (Kalyanasundaram et al 1986). In this article we review the use of Raman spectroscopy in solving problems related to colloidal semiconductors and their use in solar energy conversion.

Solar energy conversion in this present context is defined as the splitting of water to hydrogen and oxygen by sunlight in the presence of colloidal semiconductors. Electron-hole pairs created in the semiconductor by absorption of light may take part in surface redox reactions. For such redox processes to be efficient, it is important to effectively separate the electrons and holes to prevent their recombination. Therefore, relay compounds, such as methylviologen (an electron acceptor), are used to separate the photoinduced charges. The electrons formed in the semiconductor move to the surface where they are transferred to the relay species adsorbed on the surface. This electron-transfer process can be efficient only if (a) the absorption of sunlight by semiconductor is strong enough to produce a large number of electrons and holes, (b) the electron-transfer across the interface is fast enough to prevent electron-hole recombination, and (c) the relay species is adsorbed directly on the semiconductor surface. Raman spectroscopy has been used to study adsorption characteristics of the relay and electron transfer reactions at the semiconductor/ adsorbate interface.

\footnotetext{
* For correspondence
} 


\section{Raman methods}

The application of Raman spectroscopy to studies of surface adsorbed species has recently been reviewed (Bartlett and Cooney 1987; Hester 1988). Surface spectroscopies were formerly dominated by the use of ion and electron beam methods, viz. Auger electron spectroscopy, ultraviolet and X-ray photoelectron spectroscopy, electron energy loss spectroscopy and secondary ion mass spectrometry (see Clark and Hester 1988). Infrared and Raman spectroscopies provide alternative tools for probing the molecular structure of surface species. For the study of solid surfaces in contact with the aqueous phase, Raman methods are more favourable than infrared due to (a) low interference from water itself, (b) selective enhancement of vibrational modes associated with different chromophoric parts of molecules through resonance Raman methods, and (c) time-resolution into the picosecond regime.

Normal Raman scattering is an inherently weak process, with typically only one in $10^{7}$ photons resulting in Raman scattering, and therefore requires an intense light source. Although strongly scattering samples are easily amenable to normal Raman spectroscopy, one has to invoke other Raman methods such as resonance Raman (RR) or surface enhanced resonance Raman (SERR) spectroscopies for weak scatterers. RR spectroscopy and its relevance to the present work are discussed below SERR spectroscopy is not relevant to this work since worthwhile surface enhancement is observed only on certain metal surfaces, viz. mainly gold, silver and copper.

\subsection{Resonance Raman scattering}

The intensity of a Raman band associated with a transition from state $m$ to $n$ for randomly oriented molecules is given by (Clark and Dines 1986)

$$
I=K I_{0} v_{s}^{4} \sum\left|\left(\alpha_{\rho \sigma}\right)_{m n}\right|^{2}
$$

where $K$ is a constant which takes into account the permittivity of free space, $I_{0}$ is the irradiance of the incident radiation, $v_{s}$ is the scattered light frequency and $\alpha_{\rho \sigma}$ is the $\rho \sigma$ th element of the transition polarizability tensor. When the excitation frequency is in resonance with a vibronic transition from the ground state to an excited state, the transition polarizability tensor can be written as

$$
\left[\alpha_{\rho \sigma}\right]_{m n}=A+B+C+D \text {. }
$$

The terms $A, B, C$ and $D$ are responsible for different types of resonance Raman scattering. The most commonly found resonance effect due to the $A$-term is discussed here. The full description of the term involves the variable

$$
1 /\left(v_{i k}-v_{0}\right)
$$

where $v_{i k}$ is the frequency associated with the energy gap between the lowest vibrational level of the ground state and the $k$ th vibrational band of the excited electronic state, and $v_{0}$ is the incident exciting frequency. As $v_{0}$ approaches $v_{i k}$ the resonance condition is reached and the intensities of the $A$-type Raman bands increase dramatically. Thus, by selecting the appropriate excitation frequency which is in resonance with the electronic transition of a given chromophoric part of a molecule one can obtain the 
RR spectrum of that chromophore. In the present context, from the absorption spectra of the colloidal semiconductors and the adsorbed sensitizers (discussed below), RR spectra can be selectively excited from the semiconductor substrate or the sensitizer adsorbate.

\subsection{Experimental considerations}

To study the adsorption characteristics of various semiconductor/adsorbate systems, continuous wave laser excitation may be used. For the study of electron-transfer processes, time-resolved RR (TR3) methods utilising pulsed lasers may be used. A typical TR3 experiment involves a two-photon process as depicted in figure 1 . As shown, laser, L1 excites the molecule from its ground state to an excited singlet state, $E_{1}^{1}$. This may then, by a nonradiative process (for example, intersystem crossing as in the case of sensitizers to be discussed below), reach a transient triplet state, $E_{1}^{3}$. A second laser, $\mathrm{L} 2$, is used to excite the $E_{1}^{3}$ state for resonance Raman scattering. Thus, with a knowledge of lifetimes of the excited states and the rates of the non-radiative and/or the subsequent chemical processes, the RR spectra of transient species may be recorded using appropriate time delays between the pump (L1) and

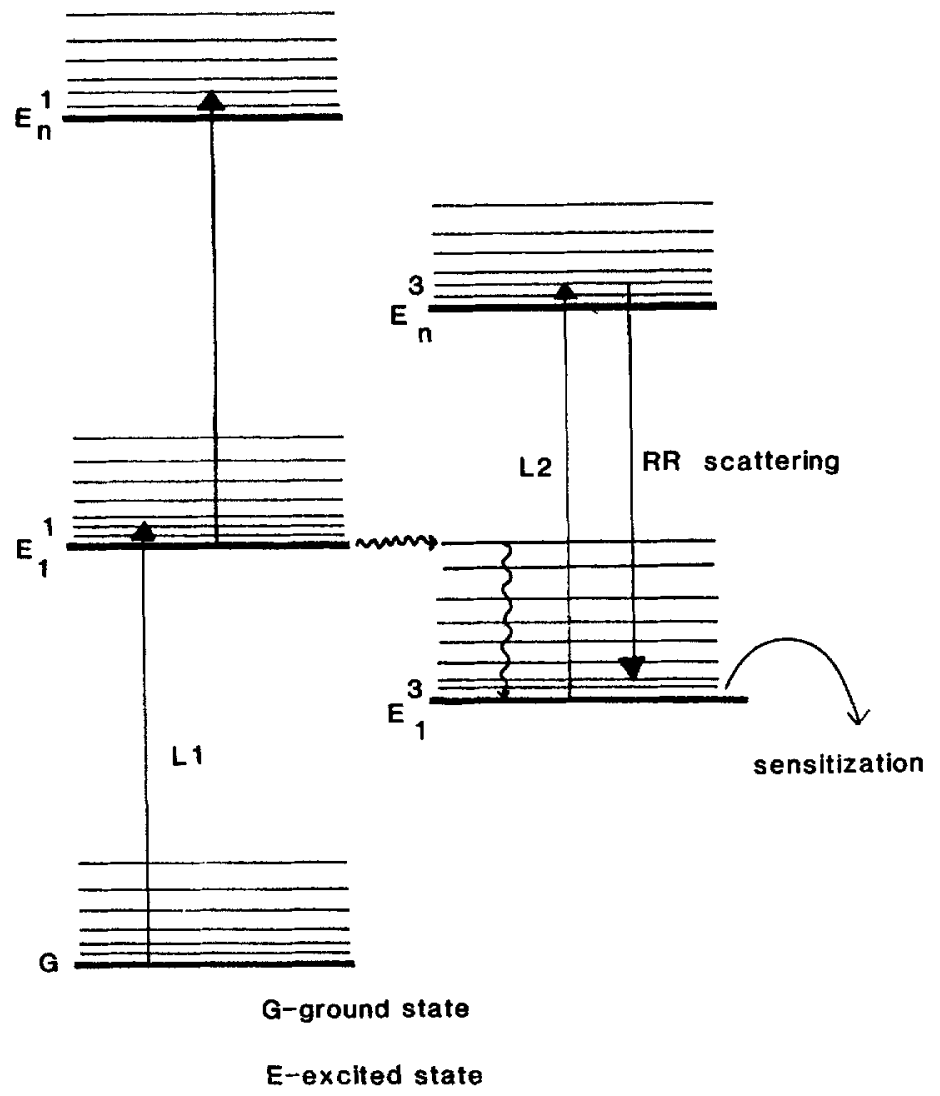

Figure 1. A multi-level schematic diagram for the dynamics involved in TR3 spectroscopy of photosensitization. 
probe (L2) lasers. With intense $\mathrm{L} 1$ radiation, multiphoton excitation to states $E_{n}^{1}$, may also result.

There are several important factors in a TR3 experiment that require careful consideration. For example, if the rate of photoexcitation to $E_{1}^{1}$ is faster than the rate of the non-radiative process, $E_{1}^{3} \leftarrow E_{1}^{1}$, then there is a competition between the induced emission from $E_{1}^{1}$ and $E_{1}^{3} \leftarrow E_{1}^{1}$. In practice, for this competition to be significant the photon flux at the sample has to be very high. Such a high flux may lead to nonlinear effects and/or dielectric breakdown of the sample. Nonlinear effects are expected to result in broad spectral bands with large frequency width at half maximum (FWHM) values (Carroll and Brus 1987a, b; Hamaguchi 1987). In order to evaluate the effects of high laser power the power-dependence of Raman spectra should be monitored. From this, one can obtain information on population saturation of a given excited state, Raman intensity saturation, and laser power threshold for sample breakdown (Bajdor et al 1987). In addition to these considerations, the details of equipment needed for such an experiment are well described in the literature (Atkinson 1982).

\section{Colloidal semiconductors as photosensitizers}

The semiconductor most used for solar energy conversion is titanium dioxide, $\mathrm{TiO}_{2}$. We take this as an example since much of our own work and earlier results have been based on this material. The optical absorption threshold of $\mathrm{TiO}_{2}$ that corresponds to the bandgap of $3.2 \mathrm{eV}$ is at $\approx 380 \mathrm{~nm}$. The solar spectrum, shown in figure 2 , has

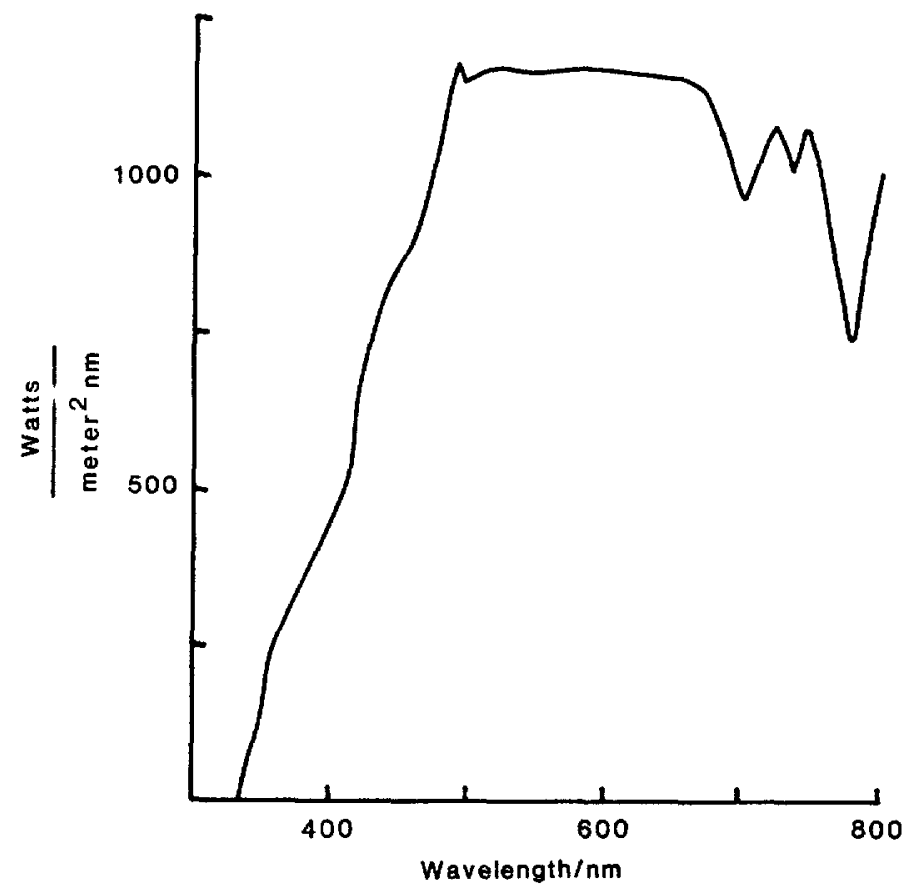

Figure 2. Solar spectrum in the visible region. 
its absorption maximum in the visible region $(400-800 \mathrm{~nm})$ and thus for $\mathrm{TiO}_{2}$ to strongly absorb sunlight, the band edge has to be shifted from $380 \mathrm{~nm}$ to longer wavelengths. This may be accomplished by surface modification of the semiconductors using inorganic complexes.

\subsection{Surface modification by secondary sensitizers}

Surface modification of colloidal semiconductors may be achieved by surface adsorption of inorganic complexes which absorb light in the visible region and are able to transfer electrons to the semiconductor from their excited states, thus acting as secondary sensitizers. The mechanism of this secondary sensitization process is depicted in figure 3. When the complex absorbs sunlight it is excited to a higher electronic state. This excited state must have a more negative reduction potential than that of the conduction band of the semiconductor, such that an electron may be injected into the conduction band from the excited state. If the complexes absorb strongly and inject electrons effectively the efficiency of the sunlight conversion to injected electrons can be high. In practice, bipyridyl complexes of ruthenium(II) have been used as secondary sensitizers with varying degrees of efficiency (Liska et al 1988). In order to improve the performance of these sensitizers, it is necessary to understand (i) the structure of these molecules in their ground and excited states as adsorbed on the semiconductor, and (ii) the nature of the electron injection process.

In this section we discuss the structure of molecules adsorbed on $\mathrm{TiO}_{2}$, and in the following section on interfacial electron-transfer reactions, the electron injection process and electron-transfer to the adsorbed relay species are discussed.

Adsorption of ruthenium(II)tris(bipyridine) (RBY) onto colloidal $\mathrm{TiO}_{2}$ surface was first investigated, since RBY has been reported to be a useful secondary sensitizer (Kalyanasundaram et al 1986). The adsorption characteristics are dependent on the Zeta potential of the colloidal semiconductor particles, which in turn depends on the $\mathrm{pH}$ of the solution. Thus positively charged RBY is expected to adsorb on a negatively charged colloidal surface, which is formed above the point of zero Zeta potential at $\mathrm{pH}=6.0$. Raman spectra were recorded from $\mathrm{RBY}$ adsorbed onto $\mathrm{TiO}_{2}$ at various $\mathrm{pH}$ values and these spectra were found to be identical to that of RBY in solution. This suggests no major perturbation to the molecular architecture due to adsorption.

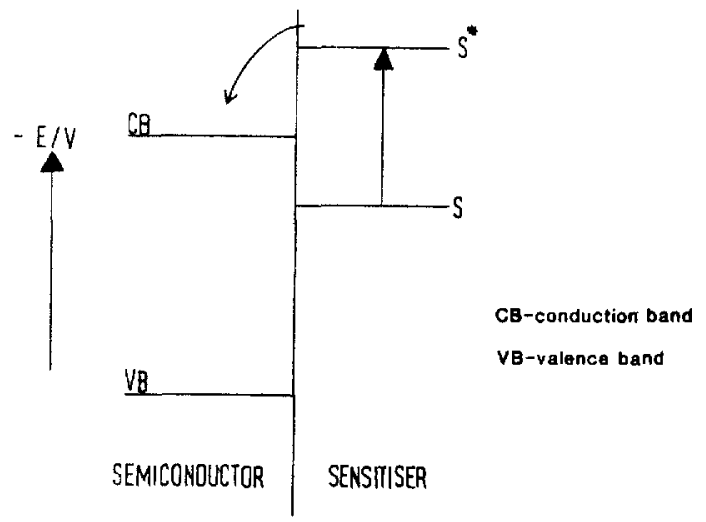

Figure 3. A schematic representation of the energetics in the sensitization process. 


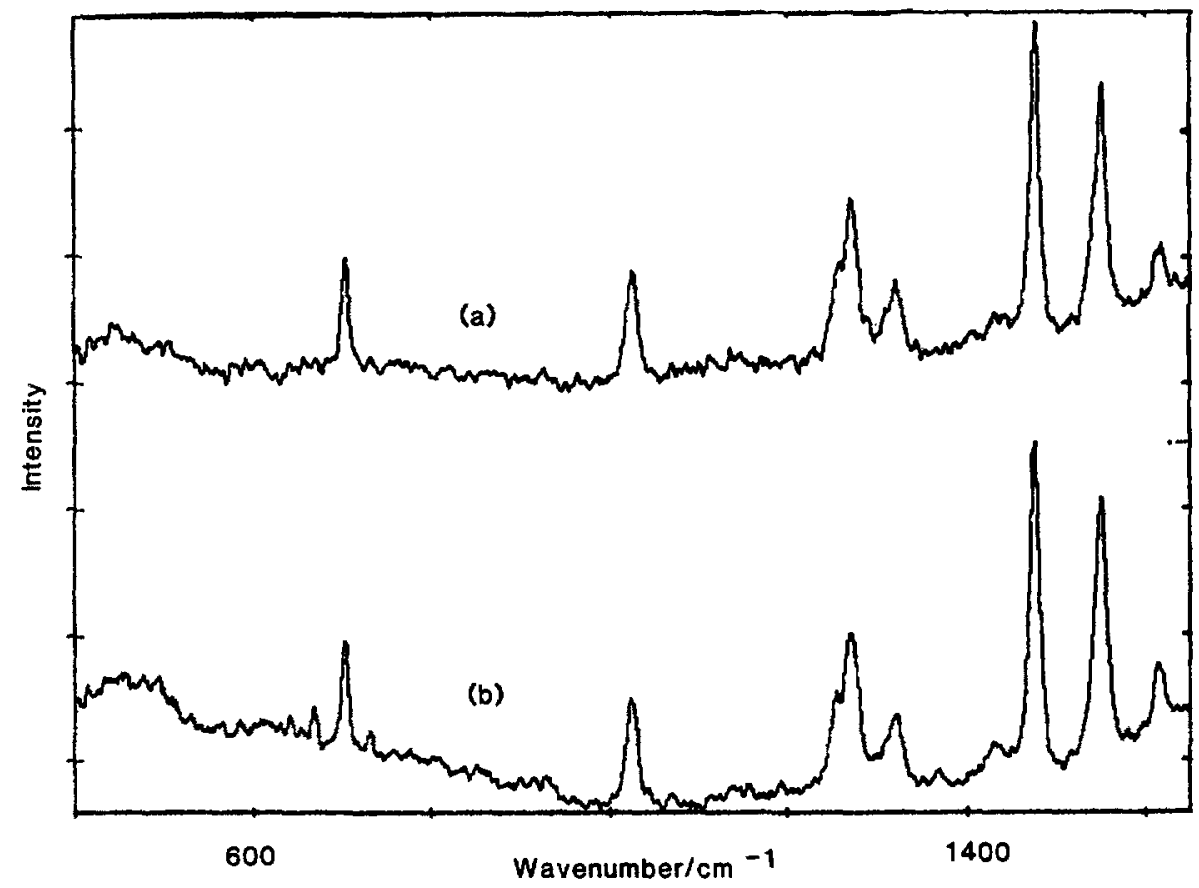

Figure 4. RR spectra of (a) $\mathrm{RB} 4 \mathrm{H}$ and (b) $\mathrm{RB} 4 \mathrm{H} / \mathrm{TiO}_{2}$ sol; $\lambda_{\text {enc }}=488 \mathrm{~nm}$ and $5 \mathrm{~cm}^{-1}$ resolution.

Similar results were also obtained for ruthenium(II)-tris(2,2'-bipyridyl-4,4'-diethyl carboxylate) (RB4ET) and ruthenium(II)-tris(2,2'-bipyridyl-4,4'-dicarboxylate) (RB4H) adsorbed on $\mathrm{TiO}_{2}$. Although $\mathrm{RB} 4 \mathrm{H}$ is reported to have an improved sensitizing efficiency compared with RBY (Desilvestro et al 1985), the RR spectra as seen in figure 4 , do not show any change in vibrational structure due to surface interactions at the colloidal semiconductor/RB4H interface. This is unlike the case of molecules adsorbed on certain metal surfaces, viz $\mathrm{Cu}, \mathrm{Ag}, \mathrm{Au}$, etc., which results in significant change in Raman band positions and intensities due to adsorption (Van Duyne 1979; Hester 1988).

Sensitization of $\mathrm{TiO}_{2}$ can be achieved using these complexes either by adsorption or by surface derivatization. In the absence of any evidence of surface interactions due to absorption, attention was focused on surface-derivatized $\mathrm{TiO}_{2}$ (Umapathy et al 1987). The RR spectrum of the surface-derivatized $\mathrm{TiO}_{2}$ was recorded (see figure 5) and an analysis of this spectrum revealed the presence of new Raman bands in the low wavenumber region due to $\mathrm{Ru}-\mathrm{O}-\mathrm{Ti}$ type surface bonding. By interpretation of this spectrum along with that of the corresponding ligand spectrum we were able to obtain evidence for the structure of $\mathrm{RB} 4 \mathrm{H}$ bound to $\mathrm{TiO}_{2}$ (Umapathy et al 1989).

Very recently, the ruthenium(II)cis-diaqua-bis (2,2'-bipyridyl-4,4'-dicarboxylate) (RBDA) complex has been successfully used to sensitize $\mathrm{TiO}_{2}$ more efficiently than $\mathrm{RB} 4 \mathrm{H}$ (Liska et al 1988). When colloidal $\mathrm{TiO}_{2}$ was mixed with RBDA at pH 4.85, the particles developed a distinct intense violet colour, while the spectrum of the supernatant liquid showed the disappearance of the complex (Liska et al 1988). The absorption spectra of $\mathrm{TiO}_{2}, \mathrm{RBDA}$ and $\mathrm{RBDA} / \mathrm{TiO}_{2}$ mixture are shown in figure 6 . 


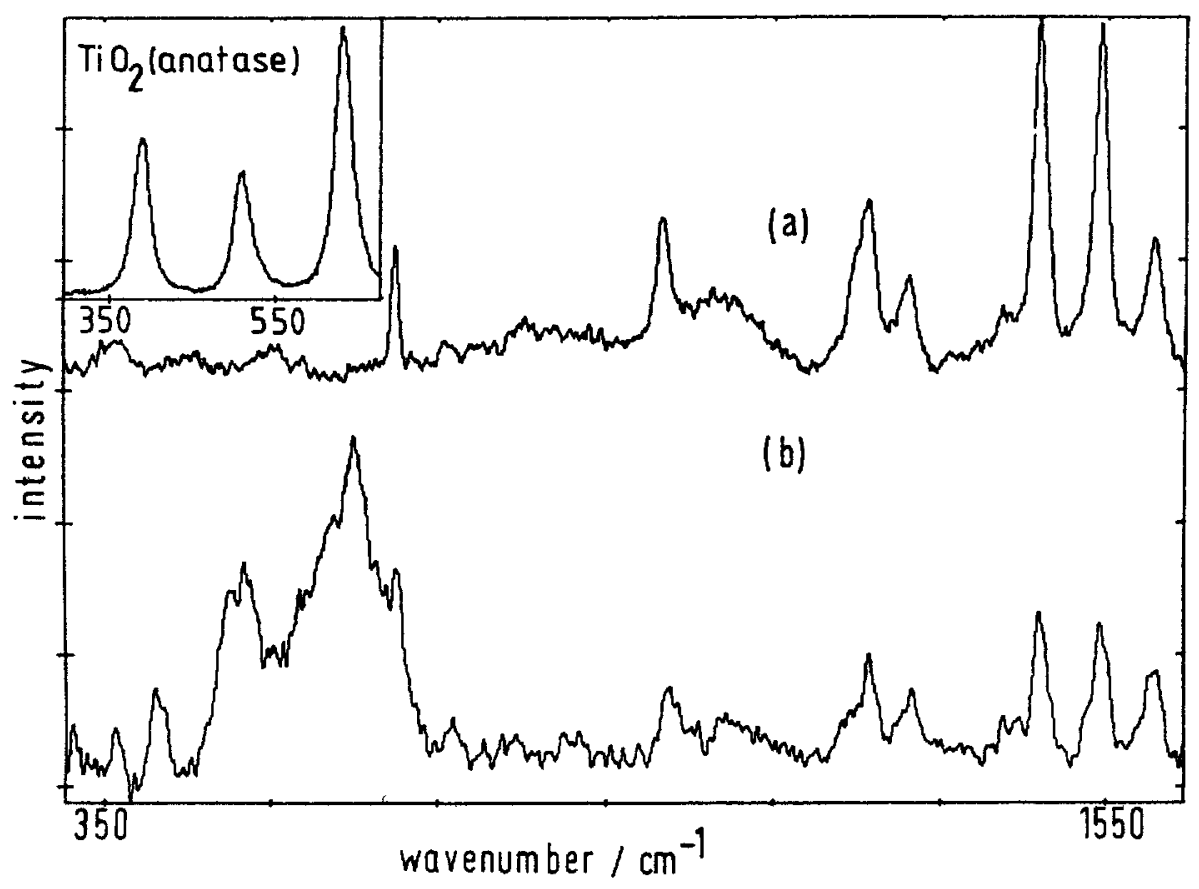

Figure 5. RR spectra of (a) RB4ET, (b) surface-derivatized RB4ET/TiO, and inset $\mathrm{TiO}_{2}$ (anatase). (Umapathy et al 1989a.)

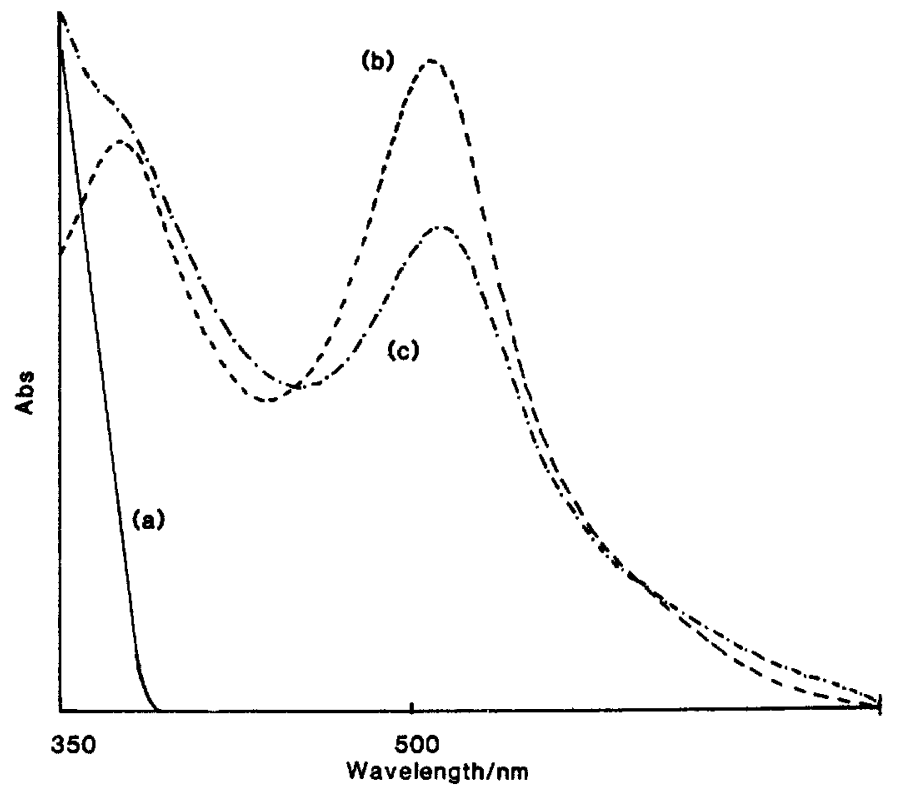

Figure 6. UV-Vis spectra of (a) $\mathrm{TiO}_{2}$ sol, (b) RBDA and (c) $\mathrm{RBDA} / \mathrm{TiO}_{2}$. 


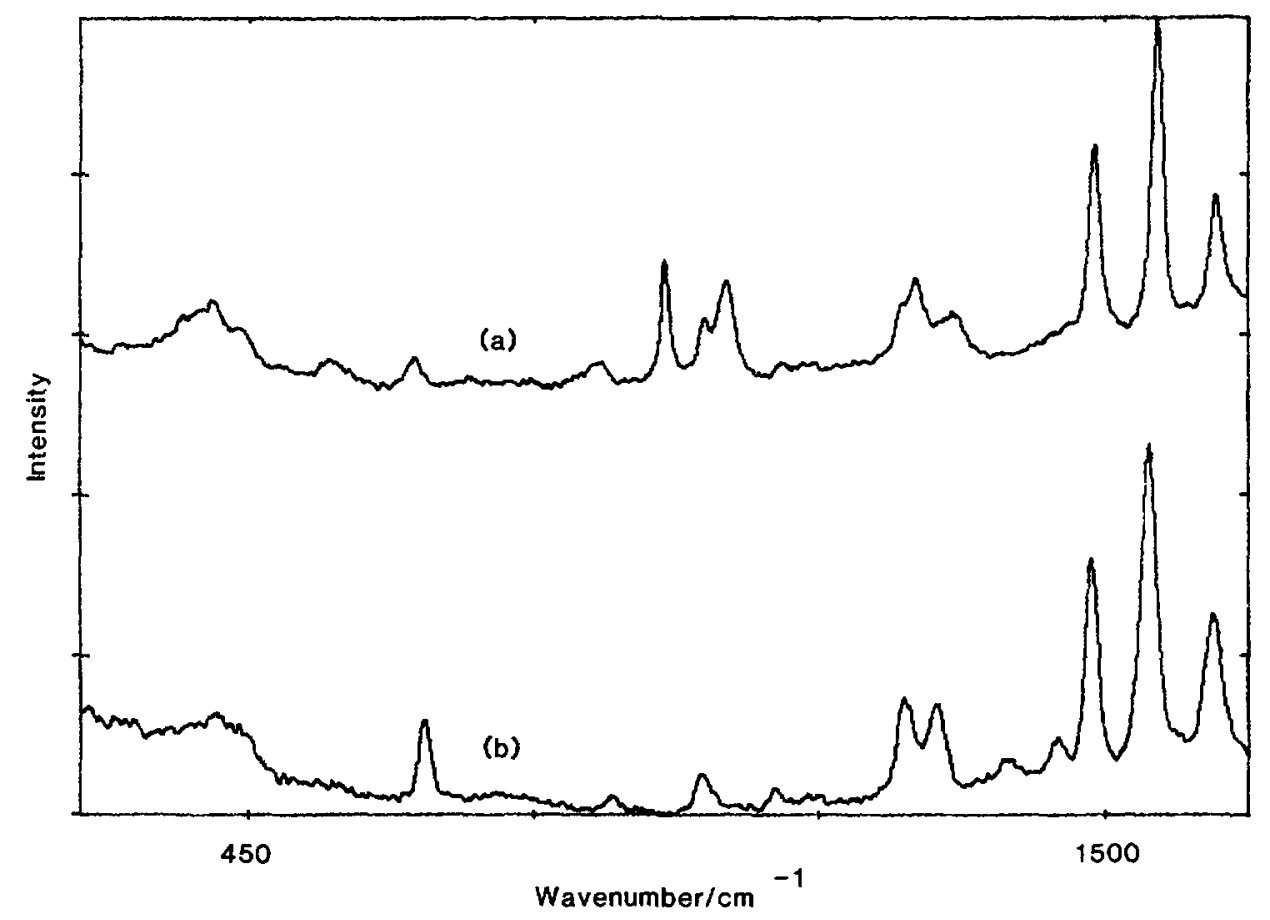

Figure 7. RR spectra of (a) RBDA and (b) $\mathrm{RBDA} / \mathrm{TiO}_{2} ; \lambda_{\mathrm{cxc}}=488 \mathrm{~nm}$ and $5 \mathrm{~cm}^{-1}$ resolution.

Figure 7 shows the RR spectra of RBDA in solution (figure 7a) and RBDA adsorbed onto $\mathrm{TiO}_{2}$ (figure 7b). Interestingly, the spectrum of $\mathrm{TiO}_{2} / \mathrm{RBDA}$ mixture differs in several respects from that of RBDA. However, there are no new Raman bands in the low wavenumber region $\left(<700 \mathrm{~cm}^{-1}\right)$ to suggest $\mathrm{Ru}-\mathrm{O}-\mathrm{Ti}$ type bonding associated with the adsorption. This result is unlike the case of either $\mathrm{RB} 4 \mathrm{H}$ adsorbed on $\mathrm{TiO}_{2}$ surface (figure 4) or surface-derivatized $\mathrm{TiO}_{2}$ (figure 5).

For an interpretation of the different Raman spectra observed for the ruthenium complexes adsorbed on $\mathrm{TiO}_{2}$, three types of adsorption are envisaged. In general, adsorption can be classified broadly into physisorption and chemisorption. We further divide physisorption into direct physisorption and solvent-separated physisorption. Chemisorption is expected to result in formation of strong covalent bonding at the surface, as observed in the case of surface-derivatized $\mathrm{TiO}_{2}$. Therefore, the appearance of new bands in the low wavenumber region (figure 5) due to $\mathrm{Ru}-\mathrm{O}$ and $\mathrm{Ti}-\mathrm{O}$ vibrations are attributed to chemisorption. In the case of direct physisorption, it is expected that surface interactions due to direct contact of the ruthenium complex and $\mathrm{TiO}_{2}$ may result in changes in their vibrational spectra (Umapathy et al 1989b). Thus the observed Raman spectra of $\mathrm{RBDA} / \mathrm{TiO}_{2}$ (figure $7 \mathrm{~b}$ ) may be attributed to the effects of direct physisorption, whereas $\mathrm{RB} 4 \mathrm{H} / \mathrm{TiO}_{2}$ (figure 4) may be explained as solvent-separated physisorption (Rossetti et al 1984). A comparison of Raman data of all these adsorbed ruthenium complexes reveals an interesting feature in the $\mathrm{RBDA} / \mathrm{TiO}_{2}$ spectrum. As recorded in table 1, the Raman band positions in the high wavenumber region ( $1000-1700 \mathrm{~cm}^{-1}$ ) of $\mathrm{RBDA} / \mathrm{TiO}_{2}$ and $\mathrm{RB} 4 \mathrm{H}$, where the bipyridyl 
Table 1. Resonance Raman band wavenumbers $\left(\mathrm{cm}^{-1}\right)$ of RBDA, RBDA/TiO and $\mathrm{RB} 4 \mathrm{H} / \mathrm{TiO}_{2}$ in water.

\begin{tabular}{lcc}
\hline RBDA & RBDA/TiO $_{2}$ & $\mathrm{RB4H} / \mathrm{TiO}_{2}$ \\
\hline 1615 & 1610 & 1605 \\
1547 & 1536 & 1538 \\
1474 & 1471 & 1479 \\
- & 1433 & - \\
1310 & 1371 & 1374 \\
- & 1296 & 1291 \\
1268 & - & 1271 \\
1254 & 1255 & - \\
1050 & - & - \\
1024 & 1021 & 1022 \\
980 & - & - \\
905 & 919 & - \\
- & 701 & 705 \\
689 & - & - \\
\hline
\end{tabular}

ligand vibrational bands are expected, are very similar. This suggests that direct physisorption of RBDA to $\mathrm{TiO}_{2}$ colloidal surface is via the aqua ligands rather than the bipyridyl ligands (Umapathy et al 1989b).

\subsection{Interfacial charge-transfer reactions}

The interfacial charge-transfer process at the semiconductor/adsorbate interface can be studied by TR3 spectroscopy. As discussed above, with the use of two lasers delayed appropriately with respect to each other, one is able to follow the changes in molecular structure that accompany the charge-transfer reactions at the interface. The interfacial reactions at a colloidal semiconductor surface are classified into two types for convenience. The first deals with sensitization by electron-injection and the second with charge-transfer to the relay species.

3.2a Sensitization by electron-injection : The first reported TR 3 study of sensitization (Rossetti and Brus 1984) involved eosin $\mathrm{Y}$ on $\mathrm{TiO}_{2}$. Eosin $\mathrm{Y}(E)$ absorbs at $515 \mathrm{~nm}$ and therefore $E$ could be photoexcited by a $532-$ nm laser pulse.

$$
\begin{aligned}
& E+h v_{532 n m} \rightarrow E^{*}, \\
& E^{*}+\mathrm{TiO}_{2} \rightarrow E^{+}+e_{\mathrm{CB}\left(\mathrm{TiO}_{2}\right)}^{-}+\mathrm{TiO}_{2} . \\
& \quad \mathrm{CB}=\text { conduction band }
\end{aligned}
$$

The excited triplet state $\left(E^{*}\right)$, formed by a fast intersystem crossing from an excited singlet state, injects an electron into the conduction band of the semiconductor. This results in the formation of oxidized eosin $\mathrm{Y}\left(E^{+}\right)$which has an absorption maximum at $\approx 460 \mathrm{~nm}$. The experiment was conducted using a two-laser set-up, involving a $532-\mathrm{nm}$ pulse to pump (photoexcite) and a 448-nm pulse to probe $E^{+}$for RR scattering. From a comparison of these spectra with that of $E^{+}$generated by triplet quenching 
experiments, the authors were able to identify the species formed as $E^{+}$. From the results obtained, it was concluded that $E^{+}$appeared with a rise time of $5 \mathrm{~ns}$ and decayed on a microsecond time scale.

We have investigated sensitization of $\mathrm{TiO}_{2}$ by $\mathrm{RB} 4 \mathrm{H}$ and $\mathrm{RB} 4 \mathrm{ET}$. The sensitization process is believed to occur by photoexcitation of the complex to its triplet state followed by electron injection to the conduction band of $\mathrm{TiO}_{2}$ (Vlachopoulos et al 1988). As with other bipyridyl complexes of ruthenium(II), the photoexcitation of RB4H by $470 \mathrm{~nm}$ into the metal-ligand charge transfer (MLCT) transition is first expected to form a singlet state, which by a fast intersystem crossing reaches an MLCT triplet state.

The excited electron in simple bipyridyl complexes of ruthenium(II) has been found to be localized on one of the ligands by Raman spectroscopy (Forster and Hester 1981; Bradley et al 1981). This excited state is therefore similar to the one-electron electrochemically reduced complex (Virdee and Hester 1984; Tait et al 1987). However, in the case of RB4H, Raman spectra of the electrochemically reduced complex provide evidence neither for the electron being localized in one of the ligands nor delocalized over all the three ligands (Donohoe et al 1986). Thus, we first investigated the excited state structure of RB4ET (which is similar to that of RB4H) using transient absorption and TR3 spectroscopy (Umapathy et al 1989b) (a) to understand the structure of the excited state involved in sensitizing $\mathrm{TiO}_{2}$ and (b) to establish whether the electron is localized or delocalized.

From transient absorption studies, the lifetime of RB4ET excited state (RB4ET*) was calculated to be $\approx 350 \mathrm{~ns}$ in air at room temperature. RB4ET* has an absorption maximum at $\approx 385 \mathrm{~nm}$. Since the ground state molecules also absorb weakly in that region, a single laser pulse can be used to both generate and probe RB4ET*. The leading part of a $390 \mathrm{~nm}$ dye laser output pulse was used to create the excited states and the trailing part of the pulse was used to generate the RR spectrum. Figure 8 shows the ground and excited state RR spectra of RB4ET and the power-dependent spectrum is shown in figure 9 . From the changes in integrated intensities of RB4H* bands with respect to reference solvent bands, we conclude that the excited triplet states for both the species are highly saturated (>90\%) (Umapathy et al 1989b). This is similar to other bipyridyl complexes of ruthenium(II) (Morris and Woodruff 1987). The shifts in band positions due to the formation of an excited state also are as expected for an electron being localized on one of the three ligands. Thus, we conclude that the excited state structure of the complex is asymmetric.

From a knowledge of the excited state structure, we have investigated the electron-transfer process from the excited triplet state to the conduction band of the semiconductor which results in the formation of the oxidized form of the complex. The rate constants and quantum yields for the processes have been determined from emission data (Desilvestro et al 1985).

$$
\begin{aligned}
& {\left[\mathrm{Ru}^{\mathrm{II}}\left(\mathrm{bpy}\left(\mathrm{COO}^{-}\right)_{2}\right)_{3}\right]^{4-}+h v_{470 \mathrm{~nm}} \rightarrow\left[\mathrm{Ru}^{\mathrm{II}}\left(\mathrm{bpy}\left(\mathrm{COO}^{-}\right)_{2}\right)_{3}\right]^{4-*},} \\
& \begin{array}{c}
{\left[\mathrm{Ru}^{\mathrm{II}}\left(\mathrm{bpy}\left(\mathrm{COO}^{-}\right)_{2}\right)_{3}\right]^{4-*}+\mathrm{TiO}_{2} \stackrel{k_{\text {laj }}}{\longrightarrow}\left[\mathrm{Ru}^{\mathrm{II}}\left(\mathrm{bpy}\left(\mathrm{COO}^{-}\right)_{2}\right)_{3}\right]^{3-}} \\
+e_{\mathrm{CB}\left(\mathrm{TiO}_{2}\right)}^{-}+\mathrm{TiO}_{2},
\end{array} \\
& k_{\mathrm{inj}}=3.2 \times 10^{7} \mathrm{~s}^{-1}, \\
& \phi_{\mathrm{inj}}=0.6,
\end{aligned}
$$




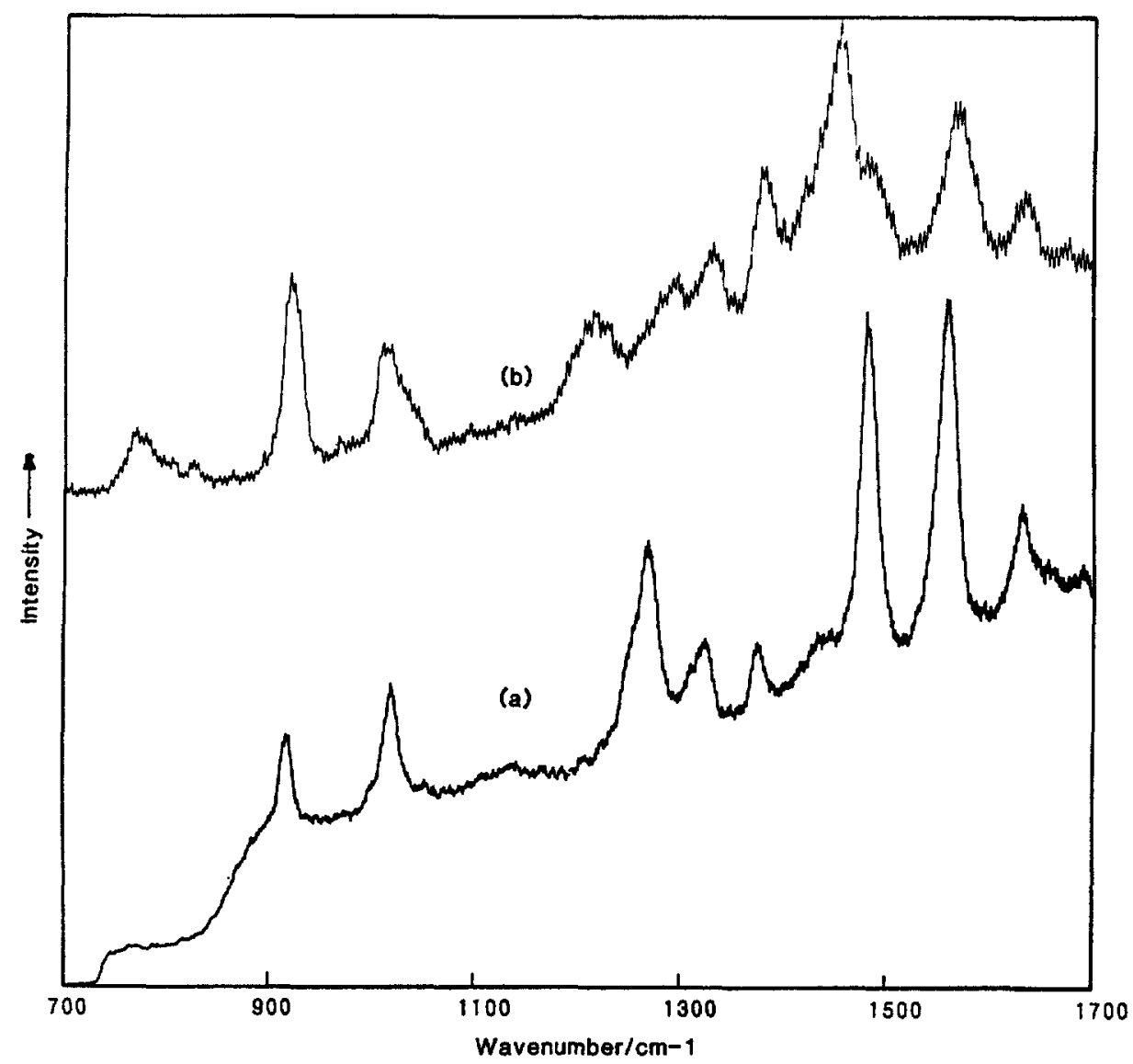

Figure 8. (a) RR spectrum of RB4ET; $\lambda_{\text {exc }}=476.5 \mathrm{~nm}$ (b) TR3 spectrum of RB4ET; $\lambda_{\mathrm{exc}}=410 \mathrm{~nm}$ and $5 \mathrm{~cm}^{-1}$ resolution.

$k_{\text {in }}$ is the calculated rate constant for electron injection into the semiconductor and $\phi_{\text {inj }}$ is the quantum yield for electron injection. The rate constant for the recapture of electrons, $k_{b}$ is calculated as $4 \times 10^{-5} \mathrm{~s}^{-1}$.

$$
\left[\mathrm{Ru}^{\mathrm{III}}\left(\mathrm{bpy}\left(\mathrm{COO}^{-}\right)_{2}\right)_{3}\right]^{3-}+e_{\mathrm{CB}\left(\mathrm{TiO}_{2}\right)}^{-} \stackrel{k_{b}}{\rightarrow}\left[\mathrm{Ru}^{\mathrm{II}}\left(\mathrm{bpy}\left(\mathrm{COO}^{-}\right)_{2}\right)_{3}\right]^{4-} \text {. }
$$

From the description of the process, it is evident that with the use of two lasers the $R R$ spectrum of the intermediate or the oxidized product $\left[\mathrm{Ru}^{\mathrm{III}}\left(\mathrm{bpy}(\mathrm{COO}-)_{2}\right)_{3}\right]^{3-}$ can be recorded.

Figure 10 shows the preliminary results obtained for this system. The pump and probe lasers were 410 and $480 \mathrm{~nm}$, respectively. The spectra obtained in the absence of the pump laser is shown in figure 10a. In the presence of a pump laser (figure 10a) and when the probe laser was delayed by $100 \mathrm{~ns}$, the RR spectra of the oxidized RB4H were recorded (figure $10 \mathrm{~b}$ ). The spectrum shows relative changes in intensities (in the $\mathrm{C}-\mathrm{C}$ and $\mathrm{C}-\mathrm{N}$ stretching vibration regions) of the bands at about 1612 and $1551 \mathrm{~cm}^{-1}$. As the delay between the pulses is increased from $100 \mathrm{~ns}$ to $1 \mathrm{~ms}$ the intensities decrease, indicating that the oxidized molecules are in the process of reverting to the 


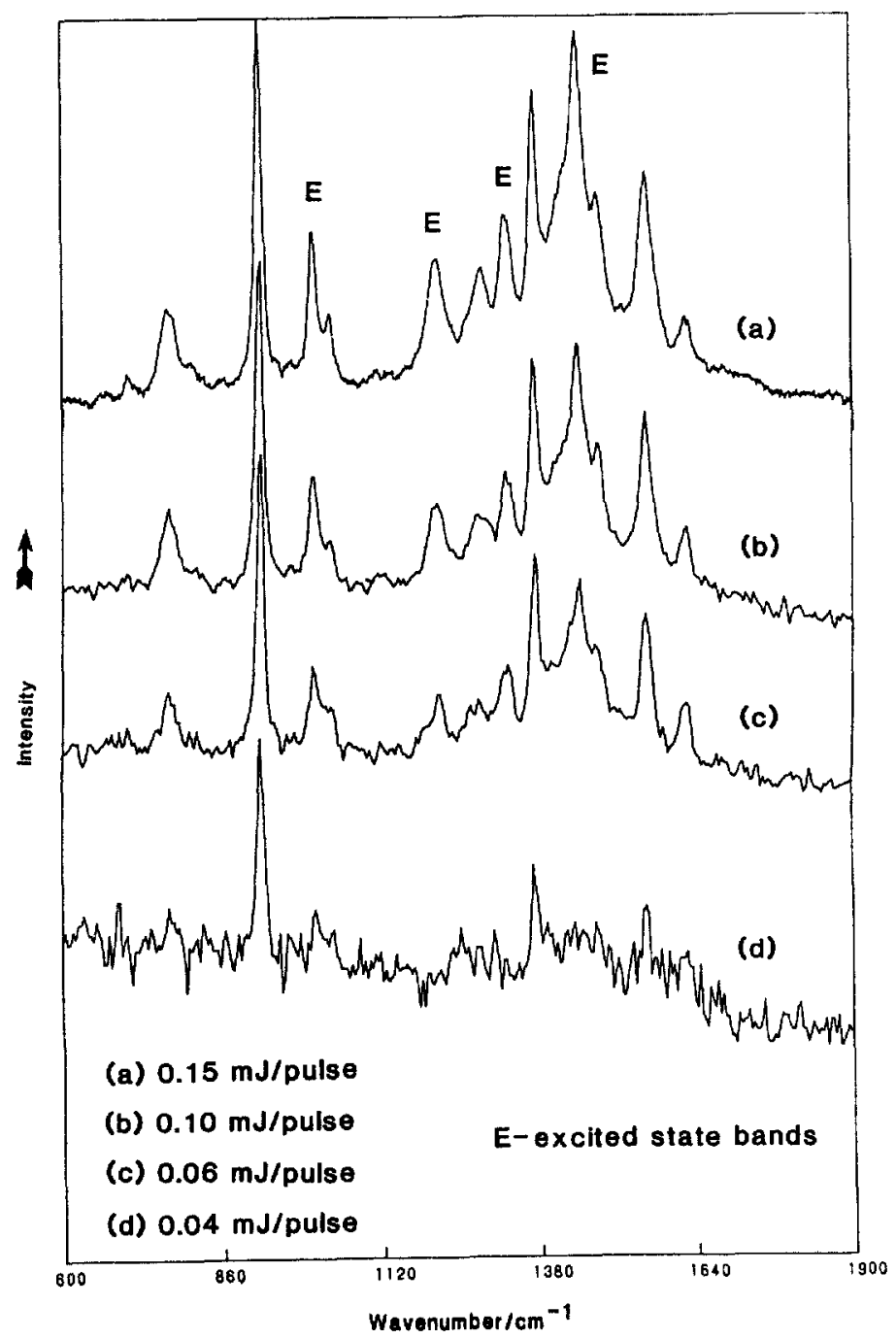

Figure 9. (a) Power dependent TR3 spectra of RB4ET; $\lambda_{\text {exc }}=410 \mathrm{~nm}$ and $5 \mathrm{~cm}^{-1}$ resolution.

original form. This is not surprising since the oxidized RB4H is expected to recapture the electrons in that time scale to form the original RB4H. These results are being analysed in more detail and the results will be published elsewhere.

3.2b Charge-transfer from semiconductors: Charge-transfer from colloidal semiconductors to surface adsorbed chemical species was studied using TR3 spectroscopy first by Brus and coworkers (Rossetti et al 1982). The processes studied were electron transfer from $\mathrm{TiO}_{2}$ to methylviologen $\left(\mathrm{MV}^{2+}\right)$ and hole transfer from $\mathrm{TiO}_{2}$ to thiocyanate $\left(\mathrm{SCN}^{-}\right)$as shown.

$$
\begin{aligned}
& \mathrm{TiO}_{2}+h v_{350 \mathrm{~nm}} \rightarrow e_{\mathrm{CB}\left(\mathrm{TiO}_{2}\right)}^{-}+h_{\mathrm{VB}\left(\mathrm{TiO}_{2}\right)}^{+}+\mathrm{TiO}_{2}, \\
& \mathrm{MV}_{\text {surf }}^{2+}+e_{\mathrm{CB}\left(\mathrm{TiO}_{2}\right)}^{-} \rightarrow \mathrm{MV}_{\text {surf }}^{+},
\end{aligned}
$$




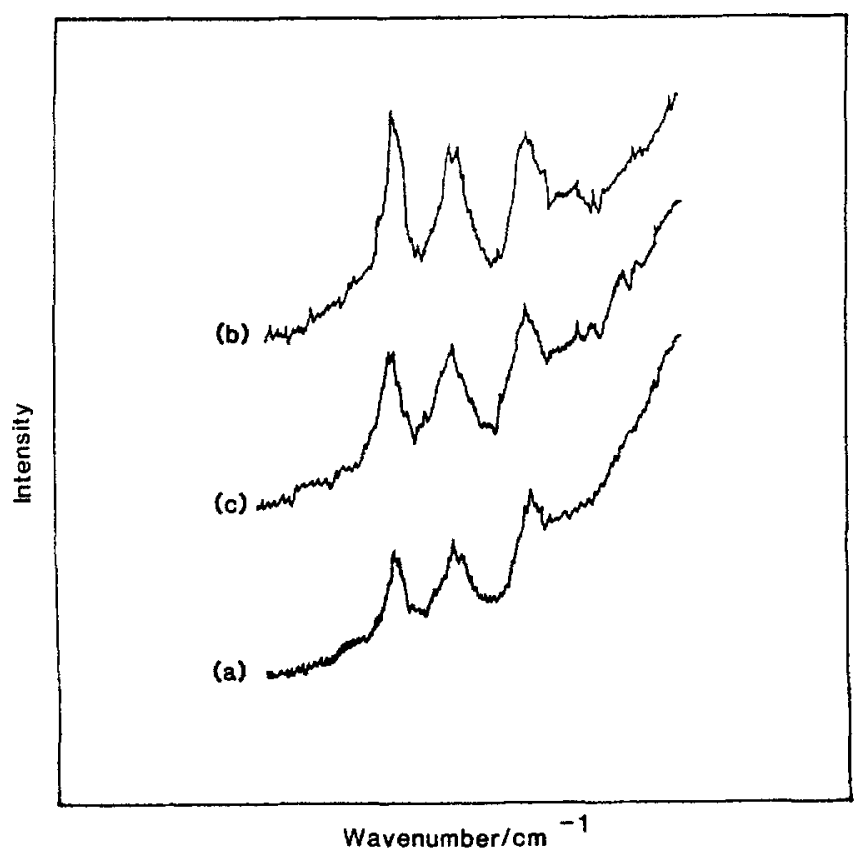

Figure 10. TR3 spectra of $\mathrm{RB} 4 \mathrm{H} / \mathrm{TiO}_{2} ; \lambda_{\text {exc/pump }}=410 \mathrm{~nm}, \lambda_{\text {exc/probe }}=480 \mathrm{~nm}$ and $5 \mathrm{~cm}^{-1}$ resolution. (a) Probe only, (b) $100 \mathrm{~ns}$ delay and (c) $1 \mathrm{~ms}$ delay.

or

$$
\mathrm{SCN}_{\text {surf }}^{-}+h_{\mathrm{VB}}^{+}\left(\mathrm{TiO}_{2}\right) \rightarrow \mathrm{SCN} \cdot \stackrel{\mathrm{SCN}^{-}}{\longrightarrow}(\mathrm{SCN})_{2 \text { surf }}^{-}
$$

CB - conduction band; VB - valence band; surf - surface adsorbed.

Here a two-laser experiment was used, one laser to pump the bandgap to produce electrons and holes and the second laser to probe the products after charge-transfer for RR scattering. The RR spectrum thus recorded for MV ${ }^{\dagger}$ was found to be identical to that of the spectrum of chemically generated $M V \pm$. Similar results were also obtained for $(\mathrm{SCN})_{2}^{-}$. A number of reasons were presented by the authors for the lack of surface perturbations. These include the possibility of diffusion of the chemical species away form the semiconductor surface after the charge-transfer process and the presence of solvation layer on the adsorbed species.

Studies on CdS colloidal particles were later reported from this laboratory (Metcalfe and Hester 1983). In this experiment a $\mathrm{CW} \mathrm{Ar}^{+}$laser was used to simultaneously photoexcite the bandgap of the semiconductor, CdS, and also probe the chargetransferred product, $\mathrm{MV}^{\ddagger} \pm$. The Raman spectrum obtained for $\mathrm{MV}^{\dagger}$ adsorbed on $\mathrm{CdS}$ again was indistinguishable from that of chemically generated $\mathrm{MV}^{+}$. These results were later confirmed independently (Rossetti et al 1984). However, picosecond TR3 methods subsequently provided evidence for $\mathrm{MV}^{+}$adsorption, from a spectrum obtained at 20 ps resolution (Rossetti and Brus 1986). The presence of broad and probably slightly shifted bands in the spectrum was attributed to directly adsorbed $\mathrm{MV}^{+}$. However, it should be borne in mind that such broadening could also arise 
from nonlinear effects, such as have been suggested for other systems in later publications from the same laboratory (Carroll and Brus 1987). Further experiments using picosecond TR3 methods are needed to clearly establish the above aspect and to understand the molecular dynamics at the colloidal surfaces. We are presently engaged in such experiments.

\section{Quantum size effects}

Quantum size effects (Q-effect) in colloidal semiconductors arise from confinement of charge carriers. This Q-effect is demonstrated for various semiconductor particles by their UV-Vis spectra, in which the shift in band gap edge towards the blue with decrease in particle size is observed. A number of theoretical studies have been published (Brus 1986, and references therein) explaining the origin of this effect and the experimental results. In this section, we briefly discuss the various factors that influence the photosensitizing properties of the colloidal semiconductors due to Q-effects.

With decrease in size, the bandgap of the semiconductors is shifted to higher energy and therefore there is a consequent change in the redox potentials (Watzke and Fendler 1987). This further leads to some interesting electron-transfer properties, as in CdSe (Nedeljkovic et al 1986). In this case bandgap irradiation of $\mathrm{CO}_{2}$-saturated solutions of dispersed colloidal CdSe particles, in sizes smaller than $50 \AA$, resulted in the formation of formic acid, whereas bulk CdSe did not generate formic acid. It has also been reported that the changes in size of colloidal particles affected the electron-transfer efficiency of the semiconductor (Watzke and Fendler 1987). In particular, small size CdS particles produce a higher yield of methylviologen radicals than large particles, due to higher electron-transfer efficiency (Watzke and Fendler 1987). Further, small $\mathrm{TiO}_{2}$ particles have been found to be photocatalytically more efficient in hydrogenation reactions than large particles (Anpo et al 1987). A number of reasons have been postulated for the increase in electron-transfer efficiency from these recent results.

These reasons include the following: (a) a decrease in particle size leads to an increase in total surface area available for molecular adsorption to aid the electrontransfer process; (b) changes in redox potentials which accompany the size changes also accelerate electron-transfer; (c) in smaller particles the charges (electrons or holes) do not have to travel a long distance before they are captured by the adsorbed species, thus avoiding electron-hole recombination and leading to more efficient electrontransfer; and (d) in smaller particles the number of surface sites with defects which assist in electron-transfer are high (Anpo et al 1987; Watzke and Fendler 1987). Although these reasons are plausible, further experiments are necessary to identify the main cause of the more efficient photosensitizing process with small semiconductor particles. To assist in this we are presently using TR3 spectroscopy to study colloidal particles, such as $\mathrm{CdS}$ and $\mathrm{TiO}_{2}$ of different sizes and their electron-transfer properties.

Raman spectroscopy has been used to study size effects in colloidal CdS semiconductors (Papavassilou 1982; Rossetti et al 1983). It has been shown that the frequencies of the Raman LO-phonon modes and the relative intensities of LO-phonon and overtone bands change with size. Recently, we have also observed that the FWHM of phonon modes of CdS increase with increase in particle size. This observation is supported by the theoretical work of Fauchet and Campbell (1988). We are presently investigating the size effect in $\mathrm{CdS}, \mathrm{InS}, \mathrm{Sb}_{2} \mathrm{~S}_{3}$ and $\mathrm{Bi}_{2} \mathrm{~S}_{3}$ using $\mathrm{RR}$ methods. 


\section{Future outlook}

RR and TR3 methods are becoming more widely available. In particular, picosecond pulsed lasers enable both the dynamics and the structures of species to be studied in the course of photoreactions. A number of unique optical properties of semiconductors of different sizes may be understood from their vibrational spectra using RR methods. Considering the importance of the Q-effect in the use of colloidal semiconductors as photosensitizers, TR3 studies of electron-transfer reactions at these small semiconductor/ adsorbate interface may be expected to yield valuable information on the dynamics and the structural characteristics of photosensitization in solar energy conversion.

\section{Acknowledgement}

We thank Ms G Lee-Son and Mr R B Girling for assistance with figures and Dr A W Parker and Ms S Tavender for technical assistance at the Rutherford Appleton Laboratory. We are grateful to the Science and Engineering Research Council for financial support.

\section{References}

Anpo M, Shima T A, Kodama S and Kubokawa Y 1987 J. Phys. Chem. 914305

Atkinson G H 1982 Advances in infrared and Raman spectroscopy (eds) R J H Clark and R E Hester (London: Heyden) vol. 9, p. 1

Bajdor, K, Nishimura Y and Peticolas W L $1987 \mathrm{~J}$. Am. Chem. Soc. 1093514

Bartlett J R and Cooney R P 1987 Advances in spectroscopy (eds) R J H Clark and R E Hester (Chichester: John Wiley) vol. 14, p. 188

Bradley P G, Kress N, Hornberger B A, Dallinger R F and Woodruff W H $1981 \mathrm{~J}$. Am. Chem. Soc. 1037441

Brus L E 1986 J. Phys. Chem. 902555

Carroll P J and Brus L E 1987a J. Chem. Phys. 866584

Carroll P J and Brus L E 1987b J. Am. Chem. Soc. 1097613

Clark R J H and Dines T J 1986 Angew. Chim. 25131

Clark R J H and Hester R E (eds) 1988 Spectroscopy of surfaces, Advances in spectroscopy (Chichester: John Wiley) vol. 16

Desilvestro J, Grätzel M, Kavan L, Moser J and Augustynski J 1985 J. Am. Chem. Soc. 1072988

Donohoe R J, Tait C D, De Armond M K and Wertz D W 1986 Spectrochim. Acta A42 233

Duonghong D, Serpone N and Grätzel M 1984 Helv. Chim. Acta 671012

Fauchet P M and Campbell I H 1988 Critical Rev. Solid State Mater. Sci. 14 S79

Forster M and Hester R E 1981 Chem. Phys. Lett. 8142

Grätzel M 1985 Photoelectrochemistry, photocatalysis and photoreactors (ed.) M Schiavello (Dordrecht: D Reidel) p. 217

Hamaguchi H 1987 Vibrational spectra and structure (ed.) J R Durig (Amsterdam: Elsevier) vol. 16, p. 227

Hercules J M 1978 Anal. Chem. 50 734A

Hester R E 1988 Comprehensive chemical kinetics (ed.) R G Compton (Amsterdam: Elsevier) vol. 28

Kalyanasundaram K, Grätzel M and Pelizzetti E 1986 Coord. Chem. Rev. 6957

Liska P, Vlachopoulos N, Nazeeruddin M K, Comte P and Grätzel M 1988 (personal communication)

Metcalfe K and Hester R E 1983 J. Chem. Soc., Chem. Commun. 133

Morris D E and Woodruff W H 1987 Advances in spectroscopy (eds) R J H Clark and R E Hester (Chichester. John Wiley) vol. 14 , p. 285

Nedeljkovic J M, Nenadovic M T, Micic O I and Nozik A J 1986 J. Phys. Chem. 9012

Papavassilou G C 1982 J. Mol. Struct. 79395

Rossetti R, Beck S M and Brus L E 1982 J. Am. Chem. Soc. 1047322

Rossetti R, Beck S M and Brus L E 1984 J. Am. Chem. Soc. 106980 
Rossetti R and Brus L E 1984 J. Am. Chem. Soc. 1064336

Rossetti R and Brus L E 1986 J. Phys. Chem. 90558

Rossetti R, Nakahara S and Brus L E 1983 J. Chem. Phys. 791086

Tait, C D, Vess T M and Wertz D W 1987 Chem. Phys. Lett. 142225

Umapathy S, Lee-Son G and Hester R E $1987 \mathrm{~J}$. Chem. Soc., Chem. Commun. 1841

Umapathy S, Lee-Son G and Hester R E 1989a J. Mol. Struct. 194107

Umapathy S, Cartner A M and Hester R E 1989b J. Phys. Chem. (in press)

Van Duyne R 1979 Chemical and biochemical applications of lasers (ed.) J B Moore (New York: Academic Press) vol. 4, p. 101

Virdee H R and Hester R E 1984 J. Phys. Chem. 88451

Vlachopoulos N, Liska P, Augustynski J and Grätzel M 1988 J. Am. Chem. Soc. 1101216

Watzke H J and Fendler J H 1987 J. Phys. Chem. 91854 